\title{
Ground states for planar axially Schrödinger-Newton system with an exponential critical growth
}

\author{
Wenbo Wang ${ }^{1}$, Quanqing $\mathrm{Li}^{2}$ and Yongkun $\mathrm{Li}^{\mathrm{i}^{*}}$
}

\author{
Correspondence: yklie@ynu.edu.cn \\ 'School of Mathematics and \\ Statistics, Yunnan University, \\ Kunming, P.R. China \\ Full list of author information is \\ available at the end of the article
}

\section{Abstract}

In this paper, we study the following planar Schrödinger-Newton system:

$$
\left\{\begin{array}{l}
-\Delta u+V(x) u+\lambda \phi u=f(x, u) \text { in } \mathbb{R}^{2} \\
\Delta \phi=u^{2} \text { in } \mathbb{R}^{2}
\end{array}\right.
$$

where $V, f$ are axially symmetric about $x, V$ is positive, and $f$ is super-linear at zero and exponential critical at infinity. Using a weaker condition

$$
\left[\frac{f(x, u)}{u^{3}}-\frac{f(x, t u)}{(t u)^{3}}\right] \operatorname{sign}(1-t)+\theta V(x) \frac{\left|1-t^{2}\right|}{(t u)^{2}} \geq 0, \quad \forall x \in \mathbb{R}^{2}, t>0, u \neq 0
$$

with $\theta \in[0,1)$ instead of the Nehari type monotonic condition on $\frac{f(x, u)}{|u|^{3}}$, we obtain a ground state solution of the above problem via variational methods.

MSC: $35 J 50 ; 35 J 20 ; 35 J 10$

Keywords: Schrödinger-Newton system; Axial symmetry; The exponential critical growth; Ground states

\section{Introduction and main results}

In the present paper, we are concerned with the wave solutions of the SchrödingerNewton system

$$
\left\{\begin{array}{l}
-i \psi_{t}-\Delta \psi+W(x) \psi+\lambda \phi u=g(x, \psi) \quad \text { in } \mathbb{R}^{d} \\
\Delta \phi=|\psi|^{2} \text { in } \mathbb{R}^{d}
\end{array}\right.
$$

where $\psi: \mathbb{R}^{d} \times \mathbb{R} \rightarrow \mathbb{C}$ is the wave function, $W(x)$ is a real external potential, $\lambda>0$ is a parameter. Problems of the type (1.1) arise in many problems from physics. We refer the readers to [15], therein (1.1) appears in a quantum mechanical context in the case $d \leq 3$.

(c) The Author(s) 2020. This article is licensed under a Creative Commons Attribution 4.0 International License, which permits use, sharing, adaptation, distribution and reproduction in any medium or format, as long as you give appropriate credit to the original author(s) and the source, provide a link to the Creative Commons licence, and indicate if changes were made. The images or other third party material in this article are included in the article's Creative Commons licence, unless indicated otherwise in a credit line to the material. If material is not included in the article's Creative Commons licence and your intended use is not permitted by statutory regulation or exceeds the permitted use, you will need to obtain permission directly from the copyright holder. To view a copy of this licence, visit http://creativecommons.org/licenses/by/4.0/ 
A standing wave solution of (1.1) is a solution of the form $\psi(x, t)=e^{-i E t} u(x)$ and its existence reduces (1.1) to the system

$$
\left\{\begin{array}{l}
-\Delta u+V(x) u+\lambda \phi u=f(x, u) \text { in } \mathbb{R}^{d}, \\
\Delta \phi=u^{2} \text { in } \mathbb{R}^{d}
\end{array}\right.
$$

where $V(x)=W(x)-E, g\left(x, e^{-i E t} u\right)=f(x, u) e^{-i E t}$. For the case $d=3$, it is called the Schrödinger-Poisson system and it has been well studied. For the existence, multiplicity, and concentration, we refer the readers to $[2,3,9,10,13,20]$ and the references therein. For Kirchhoff type equations involving subcritical and critical growth in three dimensions, please see [19] and the references therein. We also quote the paper [12] for Hardy-Schrödinger-Kirchhoff systems.

However, much less is known about the case $d=2$. For $\Delta \phi=u^{2}$, in $\mathbb{R}^{2}$, one has

$$
\phi(x)=\frac{1}{2 \pi} \int_{\mathbb{R}^{2}} \ln (|x-y|)|u(y)|^{2} d y .
$$

Substituting it into (1.2), we obtain the integro-differential equation

$$
-\Delta u+V(x) u+\frac{\lambda}{2 \pi}\left(\ln (|\cdot|) * u^{2}\right) u=f(x, u) \quad \text { in } \mathbb{R}^{2} .
$$

For simplicity, throughout this paper, let $\lambda=2 \pi$. The approach for $d=3$ cannot be easily adapted to $d=2$ since

$$
\frac{1}{4} \int_{\mathbb{R}^{2}} \int_{\mathbb{R}^{2}} \ln (|x-y|)|u(y)|^{2}|u(x)|^{2} d y d x,
$$

which is the functional associated with the third term in (1.4), is sign-changing, and is neither bounded from above nor from below on $H^{1}\left(\mathbb{R}^{2}\right)$. This difficulty has been overcome recently in [7] or [16]. For

$$
-\Delta u+\left(\ln (|\cdot|) * u^{2}\right) u=\mu u \quad \text { in } \mathbb{R}^{2}
$$

by introducing the following subspace of $H^{1}\left(\mathbb{R}^{2}\right)$

$$
X:=\left\{u \in H^{1}\left(\mathbb{R}^{2}\right): \int_{\mathbb{R}^{2}} \ln (1+|x|) u^{2} d x<\infty\right\}
$$

endowed with the norm

$$
\|u\|^{2}=\int_{\mathbb{R}^{2}}\left(|\nabla u|^{2}+u^{2}+\ln (1+|x|) u^{2}\right) d x,
$$

Stubbe considered the $L^{2}$-constraint minimization problem and proved that (1.6) admits a ground state.

Soon afterwards, in [8], Cingolani and Weth processed successfully the two dimensional Schrödinger-Newton equations with nonlinear term $|u|^{p-2} u, p \geq 4$. Du and Weth [11] provided some results about $p>2$ and $p \geq 3$. The key tool is Pohozaev type identity (see [11, Lemma 2.4]). Chen, Shi, and Tang [4] used the same idea to obtain a ground state 
but they could deal with the general nonlinearity $f(u)$. Simultaneously, Chen and Tang [5] investigated the existence of an axially symmetric Nehari type ground state and nontrivial solution for

$$
-\Delta u+V(x) u+\left(\ln (|\cdot|) * u^{2}\right) u=f(x, u) \quad \text { in } \mathbb{R}^{2},
$$

where $V, f$ is axially symmetric about $x$. Please see $[6,17]$ for further results about two dimensional Schrödinger-Newton equations with the axially symmetric assumptions. Recently, when $V(x)=1$, Alves and Figueiredo [1] proved that (1.4) admits a positive ground state, where $f$ is a continuous function with the exponential critical growth.

In this paper, motivated by the papers [1] and [5], we shall study the existence of ground state solutions of planar problem (1.1) with an exponential critical growth. In order to state our main result, we assume that

$\left(V_{1}\right) V \in C\left(\mathbb{R}^{2}, \mathbb{R}\right), \inf _{x \in \mathbb{R}^{2}} V(x)>0, V(x):=V\left(x_{1}, x_{2}\right)=V\left(\left|x_{1}\right|,\left|x_{2}\right|\right)$ for all $x \in \mathbb{R}^{2}$.

$\left(V_{2}\right)$ There exists a sequence $\left\{t_{n}\right\} \subset(0, \infty)$ such that $t_{n} \rightarrow \infty$ and

$$
\sup _{x \in \mathbb{R}^{2}, n \in \mathbb{N}} \frac{V\left(t_{n}^{-1} x\right)}{V(x)}<\infty
$$

$\left(f_{1}\right) f \in C^{1}\left(\mathbb{R}^{2} \times \mathbb{R}, \mathbb{R}\right), f(x, u):=f\left(\left(x_{1}, x_{2}\right), u\right)=f\left(\left(\left|x_{1}\right|,\left|x_{2}\right|\right), u\right)$.

$\left(f_{2}\right) f(x, u)=o(|u|)$ as $u \rightarrow 0$, uniformly in $x \in \mathbb{R}^{2}$.

$\left(f_{3}\right)$ There exists $\alpha_{0}>0$ such that

$$
\lim _{|u| \rightarrow \infty} \frac{f(x, u)}{\exp \left(\alpha u^{2}\right)}=0 \quad \text { for } \alpha>\alpha_{0}, \quad \lim _{|u| \rightarrow \infty} \frac{f(x, u)}{\exp \left(\alpha u^{2}\right)}=+\infty \quad \text { for } \alpha<\alpha_{0} .
$$

$\left(f_{4}\right)$ There exists $\theta \in[0,1)$ such that

$$
\left[\frac{f(x, \tau)}{\tau^{3}}-\frac{f(x, t \tau)}{(t \tau)^{3}}\right] \operatorname{sign}(1-t)+\theta V(x) \frac{\left|1-t^{2}\right|}{(t \tau)^{2}} \geq 0, \quad \forall x \in \mathbb{R}^{2}, t>0, \tau \neq 0 ;
$$

$\left(f_{5}\right) \inf _{x \in \mathbb{R}^{2}, u \neq 0} \frac{F(x, u)}{u^{2}}>-\infty$, where $F(u)=\int_{0}^{u} f(t) d t$.

Remark 1.1 A simple example of satisfying the hypotheses of $\left(V_{1}\right)-\left(V_{2}\right)$ is the function $V(x)=1+\left|x_{2}\right|\left[1+\sin \left(\pi\left|x_{1}\right|\right)\right]$ with $t_{n}=n$. Here we also give an example which satisfies $\left(f_{1}\right)-\left(f_{5}\right)$ :

$$
f(x, u)=\left(K(x)|u|^{3} u-V(x)|u|^{\frac{3}{2}} u+V(x)|u| u\right) \exp \left(\frac{\frac{1}{2}-\frac{\theta}{2}}{m} \pi u^{2}\right),
$$

where $K \in\left(\mathbb{R}^{2}, \mathbb{R}\right)$ is axially symmetric and $\inf _{x \in \mathbb{R}^{2}} K(x)>0, V$ satisfies $\left(V_{1}\right)$ and $\left(V_{2}\right)$. But it does not satisfy the Nehari type monotonic condition

$$
\frac{f(x, u)}{|u|^{3}} \text { is a strictly increasing function of } u \in \mathbb{R} \backslash\{0\} .
$$

Now we state our main result as follows. 
Theorem 1 For $d=2$, suppose that $\left(V_{1}\right),\left(V_{2}\right)$ and $\left(f_{1}\right)-\left(f_{5}\right)$ are satisfied. Then, for any $\alpha \in\left(0, \frac{\pi(1-\theta)}{m}\right)$, where $m$ is the least energy (it will be defined in (2.22)), $\theta$ is from $\left(f_{3}\right),(1.7)$ possesses a ground state solution.

Remark 1.2 The condition $\alpha \in\left(0, \frac{\pi(1-\theta)}{m}\right)$ is used to prove the minimizing sequence of $m$ is bounded, and please see Lemma 3.3. Up to now, we have not been able to remove it.

The paper is organized as follows. Section 2 is to establish the variational setting and to give some preliminaries. Section 3 is to prove the existence of ground states. Throughout the paper, we always assume that $\left(V_{1}\right),\left(V_{2}\right)$ and $\left(f_{1}\right)-\left(f_{5}\right)$ hold and make use of the following notations:

- $C, C_{i}(i=0,1,2, \ldots)$ for positive constants (possibly different from line to line).

- $L^{s}\left(\mathbb{R}^{2}\right):=\left\{u: \mathbb{R}^{2} \rightarrow \mathbb{R}: \int_{\mathbb{R}^{2}}|u|^{s} d x<\infty\right\}$ and $\|\cdot\|_{s}$ denotes the usual $L^{s}$-norm in $L^{s}\left(\mathbb{R}^{2}\right)$.

\section{Variational setting and preliminaries}

In this section, we begin our study by establishing the variational setting for (1.7). Let $H^{1}\left(\mathbb{R}^{2}\right)$ be the usual fractional Sobolev space with the usual norm

$$
\|u\|_{H^{1}}=\left(\int_{\mathbb{R}^{2}}\left(|\nabla u|^{2}+u^{2}\right) d x\right)^{\frac{1}{2}}
$$

and

$$
H_{\text {as }}^{1}\left(\mathbb{R}^{2}\right):=\left\{u \in H^{1}\left(\mathbb{R}^{2}\right): u(x):=u\left(x_{1}, x_{2}\right)=u\left(\left|x_{1}\right|,\left|x_{2}\right|\right), \forall x \in \mathbb{R}^{2}\right\} .
$$

By $\left(V_{1}\right)$ and $\left(f_{1}\right)$, similar to [5], let $E$ be defined as

$$
E:=\left\{u \in H_{a s}^{1}\left(\mathbb{R}^{2}\right): \int_{\mathbb{R}^{2}} V(x) u^{2} d x<\infty\right\}
$$

endowed with the norm

$$
\|u\|_{E}=\left(\int_{\mathbb{R}^{2}}\left(|\nabla u|^{2}+V(x) u^{2}+\ln (1+|x|) u^{2}\right) d x\right)^{\frac{1}{2}} .
$$

Denote

$$
\|u\|:=\left(\int_{\mathbb{R}^{2}}\left(|\nabla u|^{2}+V(x) u^{2}\right) d x\right)^{\frac{1}{2}}, \quad\|u\|_{*}:=\left(\int_{\mathbb{R}^{2}} \ln (1+|x|) u^{2} d x\right)^{\frac{1}{2}} .
$$

According to [1, Lemma 2.1], we have the following.

Proposition 2.1 $E \hookrightarrow L^{t}\left(\mathbb{R}^{2}\right)$ is compact for all $t \in[2, \infty)$.

We formally formulate problem (1.7) in a variational way as follows:

$$
\begin{aligned}
I(u)= & \frac{1}{2} \int_{\mathbb{R}^{2}}\left(|\nabla u|^{2}+V(x) u^{2}\right) d x+\frac{1}{4} \int_{\mathbb{R}^{2}} \int_{\mathbb{R}^{2}} \ln (|x-y|)|u(y)|^{2}|u(x)|^{2} d y d x \\
& -\int_{\mathbb{R}^{2}} F(x, u) d x, \quad u \in E .
\end{aligned}
$$


For simplicity of notations, denote

$$
I_{0}(u):=\int_{\mathbb{R}^{2}} \int_{\mathbb{R}^{2}} \ln (|x-y|)|u(y)|^{2}|u(x)|^{2} d y d x
$$

Similar to [8], using $\ln (r)=\ln (1+r)-\ln \left(1+\frac{1}{r}\right), \forall r>0$, it holds that

$$
\begin{aligned}
I_{0}(u)= & \int_{\mathbb{R}^{2}} \int_{\mathbb{R}^{2}} \ln (1+|x-y|)|u(y)|^{2}|u(x)|^{2} d y d x \\
& -\int_{\mathbb{R}^{2}} \int_{\mathbb{R}^{2}} \ln \left(1+\frac{1}{|x-y|}\right)|u(y)|^{2}|u(x)|^{2} d y d x \\
:= & I_{1}(u)-I_{2}(u) .
\end{aligned}
$$

We give the following proposition which is used to estimate the nonlinearity.

Proposition $2.2\left(\left[1\right.\right.$, Lemma 2.5]) For every $\alpha>0$ and for all $u \in H^{1}\left(\mathbb{R}^{2}\right)$, we have

$$
\exp \left(\alpha u^{2}\right)-1 \in L^{1}\left(\mathbb{R}^{2}\right)
$$

Moreover, if $\|\nabla u\|_{2} \leq 1,\|u\|_{2} \leq M$, and $\alpha<4 \pi$, then there exists $C>0$ independent of $u$ such that

$$
\int_{\mathbb{R}^{2}}\left[\exp \left(\alpha u^{2}\right)-1\right] d x \leq C .
$$

Lemma 2.3 $I \in C^{1}(E, \mathbb{R})$.

Proof Noting that $\ln (1+|x-y|) \leq \ln (1+|x|)-\ln (1+|y|), \forall x, y \in \mathbb{R}^{2}$, we get

$$
\left|I_{1}(u)\right| \leq 2\|u\|_{2}^{2}\|u\|_{*}^{2}
$$

In view of $\ln (1+r) \leq r, \forall r>0$, jointly with the Hardy-Littlewood-Sobolev inequality [14], we obtain

$$
\left|I_{2}(u)\right| \leq C\|u\|_{\frac{8}{3}}^{4}
$$

So $I_{0}$ is well defined in $E$.

Using $\left(f_{1}\right)-\left(f_{3}\right)$, for each $\varepsilon>0$, we have

$$
|F(x, u)| \leq \varepsilon|u|^{2}+C(\varepsilon)|u|^{p}\left[\exp \left(\alpha|u|^{2}\right)-1\right]
$$

where $p>2$. Thus, using Hölder's inequality with $s>1, \frac{1}{s}+\frac{1}{s^{\prime}}=1$, we get

$$
\begin{aligned}
\int_{\mathbb{R}^{2}} F(x, u) d x & \leq \varepsilon \int_{\mathbb{R}^{2}}|u|^{2} d x+C(\varepsilon) \int_{\mathbb{R}^{2}}|u|^{p}\left[\exp \left(\alpha|u|^{2}\right)-1\right] d x \\
& \leq \varepsilon \int_{\mathbb{R}^{2}}|u|^{2} d x+C(\varepsilon)\left(\int_{\mathbb{R}^{2}}|u|^{p s} d x\right)^{\frac{1}{s}}\left(\int_{\mathbb{R}^{2}}\left[\exp \left(s^{\prime} \alpha|u|^{2}\right)-1\right] d x\right)^{\frac{1}{s^{\prime}}} .
\end{aligned}
$$


By Propositions 2.1 and 2.2, $I$ is well defined in $E$. By [8, Lemma 2.2], $I_{0} \in C^{1}(E, \mathbb{R})$. It is easy to check that $\int_{\mathbb{R}^{2}} F(x, u) d x$ belongs to $C^{1}(E, \mathbb{R})$. Thus, $I \in C^{1}(E, \mathbb{R})$.

Based on Lemma 2.3, we have

$$
\begin{aligned}
\left\langle I^{\prime}(u), v\right\rangle= & \int_{\mathbb{R}^{2}}(\nabla u \nabla v+V(x) u v) d x+\int_{\mathbb{R}^{2}} \int_{\mathbb{R}^{2}} \ln (|x-y|)|u(y)|^{2} u(x) v(x) d y d x \\
& -\int_{\mathbb{R}^{2}} f(x, u) v d x .
\end{aligned}
$$

Lemma 2.4 For every $u \in E$, we have

$$
I(u) \geq I(t u)+\frac{1-t^{4}}{4}\left\langle I^{\prime}(u), u\right\rangle+\frac{(1-\theta)\left(1-t^{2}\right)^{2}}{4}\|u\|^{2}, \quad \forall t \geq 0 .
$$

Proof Since the proof is similar to [5, Lemma 2.3], we omit it here.

Now, we define the Nehari manifold

$$
\mathcal{N}:=\left\{u \in E \backslash\{0\}:\left\langle I^{\prime}(u), u\right\rangle=0\right\}
$$

Since the Nehari type monotonic condition on $\frac{f(x, u)}{|u|^{3}}$ and super-cubic condition are not satisfied, we need to prove that $\mathcal{N} \neq \varnothing$. To the end, we introduce the following new set:

$$
\mathcal{E}:=\left\{u \in E \backslash\{0\}: \int_{\mathbb{R}^{2}} V(x) u^{2} d x+I_{0}(u)<\int_{\mathbb{R}^{2}} f(x, u) u d x\right\} .
$$

Lemma $2.5 \mathcal{E} \neq \varnothing$.

Proof Let $u \in E$ with $u \neq 0 . u_{t}(x):=u(t x)$. By $\left(V_{2}\right)$, there exists $C_{1}>0$ such that

$$
V\left(t_{n}^{-1} x\right) \leq C_{1} V(x), \quad \forall x \in \mathbb{R}^{2}, n \in \mathbb{N}
$$

It follows that

$$
\begin{gathered}
\int_{\mathbb{R}^{2}} V(x)\left(t_{n} u_{t_{n}}\right)^{2} d x+I_{0}\left(t_{n} u_{t_{n}}\right)-\int_{\mathbb{R}^{2}} f\left(x, t_{n} u_{t_{n}}\right) t_{n} u_{t_{n}} d x \\
\leq C_{1}\|u\|^{2}+I_{0}(u)-\ln t_{n}\|u\|_{2}^{4}-\int_{\mathbb{R}^{2}} \frac{f\left(t_{n}^{-1} x, t_{n} u\right) t_{n} u}{t_{n}^{2}} d x .
\end{gathered}
$$

In view of $\left(f_{4}\right), t \geq 0, \tau \neq 0$, it holds that

$$
\begin{aligned}
& \frac{1-t^{4}}{4} \tau f(x, \tau)+F(x, t \tau)-F(x, \tau)+\frac{\theta V(x)}{4}\left(1-t^{2}\right)^{2} \tau^{2} \\
& \quad=\int_{t}^{1}\left[\frac{f(x, \tau)}{\tau^{3}}-\frac{f(x, s \tau)}{(s \tau)^{3}}\right] \operatorname{sign}(1-t)+\theta V(x) \frac{\left|1-t^{2}\right|}{(s \tau)^{2}} \tau^{3} \tau^{4} d s \geq 0 .
\end{aligned}
$$

Taking $t=0$, we obtain

$$
\frac{1}{4} \tau f(x, \tau)-F(x, \tau)+\frac{\theta V(x)}{4} \tau^{2} \geq 0, \quad \forall x \in \mathbb{R}^{2}, \tau \in \mathbb{R} .
$$


By $\left(f_{5}\right)$, one has

$$
F(x, \tau) \geq-C_{2} \tau^{2}, \quad \forall x \in \mathbb{R}^{2}, \tau \in \mathbb{R} .
$$

Thus, we get

$$
\begin{aligned}
\int_{\mathbb{R}^{2}} \frac{f\left(t_{n}^{-1} x, t_{n} u\right) t_{n} u}{t_{n}^{2}} d x & \geq \int_{\mathbb{R}^{2}}\left[\frac{4 F\left(t_{n}^{-1} x, t_{n} u\right)}{t_{n}^{2}}-\theta V\left(t_{n}^{-1} x\right) u^{2}\right] d x \\
& \geq-4 C_{2} \int_{\mathbb{R}^{2}} u^{2} d x-\theta C_{1} \int_{\mathbb{R}^{2}} V(x) u^{2} d x
\end{aligned}
$$

So

$$
\begin{aligned}
& \int_{\mathbb{R}^{2}} V(x)\left(t_{n} u_{t_{n}}\right)^{2} d x+\int_{\mathbb{R}^{2}} \int_{\mathbb{R}^{2}} \ln (|x-y|)\left|t_{n} u_{t_{n}}(y)\right|^{2}\left|t_{n} u_{t_{n}}(x)\right|^{2} d x d y \\
& -\int_{\mathbb{R}^{2}} f\left(x, t_{n} u_{t_{n}}\right) t_{n} u_{t_{n}} d x \rightarrow-\infty,
\end{aligned}
$$

which implies that $\mathcal{E} \neq \varnothing$.

The following lemma shows that $\mathcal{N} \neq \varnothing$.

Lemma 2.6 For any $u \in \mathcal{E}$, there exists unique $t>0$ such that $t u \in \mathcal{N}$.

Proof Given $u \in \mathcal{E}$, let $\gamma_{u}(t):=\left\langle I^{\prime}(t u), t u\right\rangle$ for $t>0$. Then $t u \in \mathcal{N}$ if and only if $\gamma_{u}(t)=0$. Taking $\varepsilon>0$ sufficiently small, jointly with Sobolev embedding, we obtain

$$
\begin{aligned}
\gamma_{u}(t) \geq & t^{2}\|u\|^{2}-t^{4} I_{2}(u)-\int_{\mathbb{R}^{2}} f(x, t u) t u d x \\
\geq & t^{2}\|u\|^{2}-t^{4} C_{1}\|u\|^{\frac{3}{2}}-t^{2} \varepsilon C_{2}\|u\|^{2}-t^{p} C(\varepsilon) \int_{\mathbb{R}^{2}}|u|^{p}\left[\exp \left(\alpha|t u|^{2}\right)-1\right] d x \\
\geq & t^{2}\left(1-\varepsilon C_{2}\right)\|u\|^{2}-t^{4} C_{1}\|u\|^{\frac{3}{2}} \\
& \quad-t^{p} C(\varepsilon)\left(\int_{\mathbb{R}^{2}}|u|^{s p} d x\right)^{\frac{1}{s}}\left(\int_{\mathbb{R}^{2}}\left[\exp \left(\alpha s^{\prime}\|t u\|^{2}\left(\frac{u}{\|u\|}\right)^{2}\right)-1\right] d x\right)^{\frac{1}{s^{\prime}}}
\end{aligned}
$$

Choosing $t>0$ small such that $\alpha s^{\prime}\|t u\|^{2}<4 \pi$, it follows from Proposition 2.2 that there exists $\bar{t}>0$ small enough such that

$$
\gamma_{u}(t)>0 \quad \text { for all } 0<t<\bar{t}
$$

Now, by $\left(f_{4}\right)$, one has

$$
f(x, t \tau) t \tau \geq f(x, \tau) \tau t^{4}-\theta V(x)\left(t^{2}-1\right)(t \tau)^{2}, \quad \forall x \in \mathbb{R}^{2}, t \geq 1, \tau \in \mathbb{R},
$$

which implies that

$$
\int_{\mathbb{R}^{2}}\left[\theta V(x)(t u)^{2}-f(x, t u) t u\right] d x \leq t^{4} \int_{\mathbb{R}^{2}}\left[\theta V(x) u^{2}-f(x, u) u\right] d x, \quad \forall t \geq 1
$$


Therefore,

$$
\begin{aligned}
\gamma_{u}(t)= & t^{2}\|u\|^{2}+t^{4} I_{0}(u)-\int_{\mathbb{R}^{2}} f(x, t u) t u d x \\
\leq & t^{2}\|u\|^{2}+t^{4}\left[\int_{\mathbb{R}^{2}}\left[V(x) u^{2}-f(x, u) u\right] d x+I_{0}(u)\right] \\
& -\theta t^{2} \int_{\mathbb{R}^{2}} V(x) u^{2} d x, \quad \forall t \geq 1 .
\end{aligned}
$$

Thus, we have $\gamma_{u}(t) \rightarrow-\infty$, as $t \rightarrow \infty$. So there exists $t_{0}>0$ such that $\gamma_{u}\left(t_{0}\right)=0$. Next, we shall prove that $t_{0}$ is unique. Suppose to the contrary that there are $t_{1}, t_{2}>0$ with $t_{1} \neq t_{2}$ such that $\gamma_{u}\left(t_{1}\right)=\gamma_{u}\left(t_{2}\right)=0$. For $t_{1} u \in E$, using Lemma 2.4, for all $t>0$, we have

$$
I\left(t_{1} u\right) \geq I\left(t t_{1} u\right)+\frac{(1-\theta)\left(1-t^{2}\right)^{2} t_{1}^{2}\|u\|^{2}}{4} .
$$

Taking $t=\frac{t_{2}}{t_{1}}$, it yields that

$$
I\left(t_{1} u\right) \geq I\left(t_{2} u\right)+\frac{(1-\theta)\left(1-\left(\frac{t_{2}}{t_{1}}\right)^{2}\right)^{2} t_{1}^{2}\|u\|^{2}}{4} .
$$

Similarly, one has

$$
I\left(t_{2} u\right) \geq I\left(t_{1} u\right)+\frac{(1-\theta)\left(1-\left(\frac{t_{1}}{t_{2}}\right)^{2}\right)^{2} t_{2}^{2}\|u\|^{2}}{4} .
$$

We obtain $t_{1}=t_{2}$, so it is absurd.

Since $u \in \mathcal{N}$, by Lemma 2.4 , one has

$$
I(u)=I(u)-\frac{1}{4}\left\langle I^{\prime}(u), u\right\rangle \geq \frac{1-\theta}{4}\|u\|^{2} .
$$

So we can define

$$
m:=\inf _{u \in \mathcal{N}} I(u)
$$

Up to this stage, preparations have been made. We point out that we can define $m$ without using the condition $\alpha \in\left(0, \frac{\pi(1-\theta)}{m}\right)$. In the next section, taking full advantage of the condition $\alpha \in\left(0, \frac{\pi(1-\theta)}{m}\right)$, we shall prove the existence of ground state solutions of (1.7).

\section{Existence of ground states}

In this section, with the additional condition $\alpha \in\left(0, \frac{\pi(1-\theta)}{m}\right)$, we are devoted to showing that $m$ is achieved and the minimizer is a ground state solution of equation (1.7).

Lemma 3.1 There exists $C>0$ such that $\|u\| \geq C$ for all $u \in \mathcal{N}$; furthermore, $m>0$.

Proof Assume by contradiction that there is $\left\{u_{n}\right\} \subset \mathcal{N}$ such that $\left\|u_{n}\right\| \rightarrow 0$. Obviously,

$$
\left\|u_{n}\right\|^{2}+4\left\langle I_{1}^{\prime}\left(u_{n}\right), u_{n}\right\rangle=4\left\langle I_{2}^{\prime}\left(u_{n}\right), u_{n}\right\rangle+\int_{\mathbb{R}^{2}} f\left(x, u_{n}\right) u_{n} d x
$$


In view of $\left(f_{1}\right)-\left(f_{3}\right)$, combining Hölder's inequality, it follows that

$$
\begin{aligned}
& \left|\int_{\mathbb{R}^{2}} f\left(x, u_{n}\right) u_{n} d x\right| \\
& \quad \leq \varepsilon \int_{\mathbb{R}^{2}}\left|u_{n}\right|^{2} d x+C(\varepsilon) \int_{\mathbb{R}^{2}}\left|u_{n}\right|^{p}\left[\exp \left(\alpha\left|u_{n}\right|^{2}\right)-1\right] d x \\
& \quad \leq \varepsilon \int_{\mathbb{R}^{2}}\left|u_{n}\right|^{2} d x \\
& \quad+C(\varepsilon)\left(\int_{\mathbb{R}^{2}}\left|u_{n}\right|^{s p} d x\right)^{\frac{1}{s}}\left(\int_{\mathbb{R}^{2}}\left[\exp \left(\alpha s^{\prime}\left\|u_{n}\right\|^{2}\left(\frac{u_{n}}{\left\|u_{n}\right\|}\right)^{2}\right)-1\right] d x\right)^{\frac{1}{s^{\prime}}} .
\end{aligned}
$$

With Proposition 2.2 in hand, using the Sobolev embedding, it leads to

$$
\int_{\mathbb{R}^{2}} f\left(x, u_{n}\right) u_{n} d x=o_{n}(1)
$$

By direct calculation, it holds that

$$
4\left\langle I_{2}^{\prime}\left(u_{n}\right), u_{n}\right\rangle \leq C\left\|u_{n}\right\|_{\frac{8}{3}}^{4}=o_{n}(1) .
$$

Thus, one has

$$
\left\langle I_{1}^{\prime}\left(u_{n}\right), u_{n}\right\rangle=o_{n}(1) \text {. }
$$

Therefore, we obtain

$$
\begin{aligned}
\left\|u_{n}\right\|^{2} & \leq 4\left|I_{1}^{\prime}\left(u_{n}\right), u_{n}\right\rangle+\int_{\mathbb{R}^{2}} f\left(x, u_{n}\right) u_{n} d x \\
& \leq o_{n}(1)+\varepsilon \int_{\mathbb{R}^{2}}\left|u_{n}\right|^{2} d x+C(\varepsilon) \int_{\mathbb{R}^{2}}\left|u_{n}\right|^{p}\left[\exp \left(\alpha\left|u_{n}\right|^{2}\right)-1\right] d x .
\end{aligned}
$$

That is,

$$
\begin{aligned}
& (1-\varepsilon C)\left\|u_{n}\right\|^{2} \\
& \quad \leq o_{n}(1)+C(\varepsilon)\left(\int_{\mathbb{R}^{2}}\left|u_{n}\right|^{s p} d x\right)^{\frac{1}{s}}\left(\int_{\mathbb{R}^{2}}\left[\exp \left(\alpha s^{\prime}\left\|u_{n}\right\|^{2}\left(\frac{u_{n}}{\left\|u_{n}\right\|}\right)^{2}\right)-1\right] d x\right)^{\frac{1}{s^{\prime}}} .
\end{aligned}
$$

Noting that $\left\|u_{n}\right\| \rightarrow 0$, using Proposition 2.2 again, we get

$$
(1-\varepsilon C)\left\|u_{n}\right\|^{2} \leq C(\varepsilon)\left\|u_{n}\right\|^{p},
$$

which is ridiculous. Combining with (2.21), we have $m>0$.

Next, we give the following lemma which shall be used later.

Lemma 3.2 For every $u \in E$, it holds that $I_{1}(u) \geq \frac{1}{16}\|u\|_{2}^{2}\|u\|_{*}^{2}$. 
Proof The proof is similar to [5, Lemma 2.2]. Let

$$
\Lambda_{1}:=\left\{\left(x_{1}, x_{2}\right) \in \mathbb{R}^{2}, x_{1}>0, x_{2} \geq 0\right\}, \quad \Lambda_{3}:=\left\{\left(x_{1}, x_{2}\right) \in \mathbb{R}^{2}, x_{1}<0, x_{2} \leq 0\right\} .
$$

For any $(x, y) \in \Lambda_{1} \times \Lambda_{3}$, it holds that

$$
|x-y|=\sqrt{|x|^{2}+|y|^{2}-2 x \cdot y} \geq \sqrt{|x|^{2}+|y|^{2}} \geq|x| .
$$

Thus,

$$
\begin{aligned}
I_{1}(u) & =\int_{\mathbb{R}^{2}} \int_{\mathbb{R}^{2}} \ln (1+|x-y|)|u(y)|^{2}|u(x)|^{2} d y d x \\
& \geq \int_{\Lambda_{3}} \int_{\Lambda_{1}} \ln (1+|x-y|)|u(y)|^{2}|u(x)|^{2} d y d x \\
& \geq \int_{\Lambda_{3}}|u(y)|^{2} d y \int_{\Lambda_{1}} \ln (1+|x|)|u(x)|^{2} d x \\
& =\frac{1}{16}\|u\|_{2}^{2}\|u\|_{*}^{2} .
\end{aligned}
$$

Let $\left\{u_{n}\right\} \subset \mathcal{N}$ be a minimizing sequence of $m$. On the additional condition $\alpha \in\left(0, \frac{\pi(1-\theta)}{m}\right)$, we want to prove that $\left\{u_{n}\right\}$ is bounded in $E$.

Lemma 3.3 If $\alpha \in\left(0, \frac{\pi(1-\theta)}{m}\right)$, we have $\left\{u_{n}\right\}$ is bounded in $E$.

Proof Similar to (2.21), $\left\{\left\|u_{n}\right\|\right\}$ is bounded. Similar to (2.5), $\left\{I_{2}\left(u_{n}\right)\right\}$ is bounded. Next, we want to estimate the $\left\{I_{1}\left(u_{n}\right)\right\}$. Note that

$$
\left|\int_{\mathbb{R}^{2}} f\left(x, u_{n}\right) u_{n} d x\right| \leq \varepsilon \int_{\mathbb{R}^{2}}\left|u_{n}\right|^{2} d x+C(\varepsilon) \int_{\mathbb{R}^{2}}\left|u_{n}\right|^{p}\left[\exp \left(\alpha\left|u_{n}\right|^{2}\right)-1\right] d x
$$

For the second term on the right, using Hölder's inequality with $s^{\prime}>1$ and $s^{\prime} \approx 1$, it holds that

$$
\begin{aligned}
& \int_{\mathbb{R}^{2}}\left|u_{n}\right|^{p}\left[\exp \left(\alpha\left|u_{n}\right|^{2}\right)-1\right] d x \\
& \quad \leq\left(\int_{\mathbb{R}^{2}}\left|u_{n}\right|^{s p} d x\right)^{\frac{1}{s}}\left(\int_{\mathbb{R}^{2}}\left[\exp \left(\alpha s^{\prime}\left\|u_{n}\right\|^{2}\left(\frac{u_{n}}{\left\|u_{n}\right\|}\right)^{2}\right)-1\right] d x\right)^{\frac{1}{s^{\prime}}} .
\end{aligned}
$$

Taking into account $\alpha \in\left(0, \frac{\pi(1-\theta)}{m}\right)$, jointly with

$$
\frac{1-\theta}{4}\left\|u_{n}\right\|^{2} \leq I\left(u_{n}\right) \rightarrow m
$$

for $n$ large enough, we obtain $\alpha s^{\prime}\left\|u_{n}\right\|^{2}<4 \pi$. So, by Proposition 2.2, we get

$$
\left|\int_{\mathbb{R}^{2}} f\left(x, u_{n}\right) u_{n} d x\right| \leq \varepsilon \int_{\mathbb{R}^{2}}\left|u_{n}\right|^{2} d x+C(\varepsilon) C\left(\int_{\mathbb{R}^{2}}\left|u_{n}\right|^{s p} d x\right)^{\frac{1}{s}}
$$


Since

$$
\left\|u_{n}\right\|^{2}+I_{1}\left(u_{n}\right)=I_{2}\left(u_{n}\right)+\int_{\mathbb{R}^{2}} f\left(x, u_{n}\right) u_{n} d x,
$$

which yields that $\left\{I_{1}\left(u_{n}\right)\right\}$ is bounded. And it follows from Lemma 3.2 that $\left\{u_{n}\right\}$ is bounded in $E$.

Next, we claim that there are $R, \eta>0$ such that

$$
\liminf _{n \rightarrow \infty} \int_{B_{R}\left(y_{n}\right)}\left|u_{n}\right|^{2} d x \geq \eta
$$

If it is false, using Lions' lemma (see [18, Lemma 1.21]), we get $u_{n} \rightarrow 0$ in $L^{t}\left(\mathbb{R}^{2}\right)$ for all $t \in[2, \infty)$. Noting that

$$
\left|I_{1}\left(u_{n}\right)\right| \leq 2\left\|u_{u}\right\|_{2}^{2}\left\|u_{n}\right\|_{*}^{2}=o_{n}(1), \quad\left|I_{2}\left(u_{n}\right)\right| \leq C\left\|u_{n}\right\|_{\frac{8}{3}}^{4}=o_{n}(1),
$$

similar to (3.5), it holds that

$$
\begin{aligned}
\left\|u_{n}\right\|^{2} & =o_{n}(1)+\int_{\mathbb{R}^{2}} f\left(x, u_{n}\right) u_{n} d x \\
& \leq o_{n}(1)+\varepsilon \int_{\mathbb{R}^{2}}\left|u_{n}\right|^{2} d x+C(\varepsilon) C\left(\int_{\mathbb{R}^{2}}\left|u_{n}\right|^{s p} d x\right)^{\frac{1}{s}} \\
& =o_{n}(1),
\end{aligned}
$$

which contradicts Lemma 3.1.

Lemma 3.4 $m$ is achieved and the minimizer is a weak solution of (1.7).

Proof Now, we can assume that $u_{n} \rightarrow u_{0} \neq 0$ in $E, u_{n} \rightarrow u_{0}$ in $L^{t}\left(\mathbb{R}^{2}\right)$ for all $t \in[2, \infty)$ and $u_{n}(x) \rightarrow u_{0}(x)$ a.e. in $\mathbb{R}^{2}$. By a standard argument, one can deduce that $I^{\prime}\left(u_{0}\right)=0$. Obviously, we have

$$
\begin{aligned}
& \int_{\mathbb{R}^{2}} F\left(x, u_{n}\right) d x=\int_{\mathbb{R}^{2}} F\left(x, u_{0}\right) d x+o_{n}(1), \\
& \int_{\mathbb{R}^{2}} f\left(x, u_{n}\right) u_{n} d x=\int_{\mathbb{R}^{2}} f\left(x, u_{0}\right) u_{0} d x+o_{n}(1) .
\end{aligned}
$$

Here, we only check 3.12 since (3.11) is similar. We have already known that

$$
\left|f\left(x, u_{n}\right) u_{n}\right| \leq \varepsilon\left|u_{n}\right|^{2}+C(\varepsilon)\left|u_{n}\right|^{p}\left[\exp \left(\alpha\left\|u_{n}\right\|^{2}\left(\frac{u_{n}}{\left\|u_{n}\right\|}\right)^{2}\right)-1\right]
$$

Noting that $\alpha \in\left(0, \frac{\pi(1-\theta)}{m}\right)$ and (3.5), we obtain that $\alpha\left\|u_{n}\right\|^{2}<4 \pi$ for $n$ large enough. By Proposition 2.2, there exists $C>0$ independent of $n$ such that

$$
\int_{\mathbb{R}^{2}}\left[\exp \left(\alpha\left\|u_{n}\right\|^{2}\left(\frac{u_{n}}{\left\|u_{n}\right\|}\right)^{2}\right)-1\right] d x \leq C .
$$


It follows from [18, Lemma A.1] and Lebesgue dominated convergence theorem that

$$
\lim _{n \rightarrow \infty} \int_{\mathbb{R}^{2}} f\left(x, u_{n}\right) u_{n} d x=\int_{\mathbb{R}^{2}} f\left(x, u_{0}\right) u_{0} d x
$$

Thus, we have

$$
\begin{aligned}
m & =\lim _{n \rightarrow \infty}\left[I\left(u_{n}\right)-\frac{1}{4}\left\langle I^{\prime}\left(u_{n}\right), u_{n}\right\rangle\right] \\
& \geq \frac{1}{4}\left\|u_{0}\right\|^{2}+\int_{\mathbb{R}^{2}}\left[\frac{1}{4} f\left(x, u_{0}\right)-F\left(x, u_{0}\right)\right] d x \\
& =I\left(u_{0}\right)-\frac{1}{4}\left\langle I^{\prime}\left(u_{0}\right), u_{0}\right\rangle \\
& \geq m .
\end{aligned}
$$

\section{Acknowledgements}

The authors are grateful to the reviewer for her/his valuable comments upon which the paper was revised.

\section{Funding}

This work is supported by the National Natural Science Foundation of China (11901514, 11861072, 11801153), the Honghe University Doctoral Research Programs (XJ17B11), the Yunnan Province Applied Basic Research for Youths (2018FD085), the Yunnan Province Local University (Part) Basic Research Joint Project (2017FH001-013), and the Yunnan Province Applied Basic Research for General Project (2019FB001).

\section{Abbreviations}

Not applicable.

\section{Availability of data and materials}

Not applicable.

\section{Competing interests}

The authors declare that they have no competing interests.

\section{Consent for publication}

All authors agree to publish this paper to Boundary Value Problems.

\section{Authors' contributions}

Each of the authors contributed to each part of this study equally, all authors read and approved the final manuscript.

\section{Author details}

'School of Mathematics and Statistics, Yunnan University, Kunming, P.R. China. ${ }^{2}$ Department of Mathematics, Honghe University, Mengzi, P.R. China.

\section{Publisher's Note}

Springer Nature remains neutral with regard to jurisdictional claims in published maps and institutional affiliations.

Received: 31 December 2019 Accepted: 27 February 2020 Published online: 05 March 2020

\section{References}

1. Alves, C.O., Figueieredo, G.M.: Existence of positive solution for a planar Schrödinger-Poisson system with exponential growth. J. Math. Phys. 60, Article ID 011503 (2019)

2. Azzollini, A., d'Avenia, P., Pomponio, A.: On the Schrödinger-Maxwell equations under the effect of a general nonlinear term. Ann. Inst. Henri Poincaré, Anal. Non Linéaire 27, 779-791 (2010)

3. Cerami, G., Vaira, G.: Positive solutions for some non-autonomous Schrödinger-Poisson systems. J. Differ. Equ. 248 $521-543(2010)$

4. Chen, S., Shi, J., Tang, X.: Ground state solutions of Nehari-Pohozaev type for the planar Schrödinger-Poisson system with general nonlinearity. Discrete Contin. Dyn. Syst. 39, 5867-5889 (2019)

5. Chen, S., Tang, X.: Existence of ground state solutions for the planar axially symmetric Schrödinger-Poisson system. Discrete Contin. Dyn. Syst., Ser. B 24(9), 4685-4702 (2019)

6. Chen, S., Tang, X.: On the planar Schrödinger-Poisson system with the axially symmetric potential. J. Differ. Equ. 268 945-976 (2019)

7. Choquard, P., Stubbe, J., Vuffray, M.: Stationary solutions of the Schrödinger Newton model an ODE approach. Differ. Integral Equ. 21, 665-679 (2008) 
8. Cingolani, S., Weth, T.: On the Schrödinger-Poisson system. Ann. Inst. Henri Poincaré, Anal. Non Linéaire 33, 169-197 (2016)

9. Coclite, G.M.: A multiplicity result for the nonlinear Schrödinger-Maxwell equations. Commun. Appl. Anal. 7, 417-423 (2003)

10. d'Avenia, P.: Non-radially symmetric solutions of nonlinear Schrödinger equation coupled with Maxwell equations. Adv. Nonlinear Stud. 2,177-192 (2002)

11. Du, M., Weth, T.: Ground states and high energy solutions of the planar Schrödinger-Poisson system. Nonlinearity 30, 3492-3515 (2017)

12. Fiscella, A., Pucci, P., Zhang, B.: $p$-Fractional Hardy-Schrödinger-Kirchhoff systems with critical nonlinearities. Adv. Nonlinear Anal. 8(1), 1111-1131 (2019)

13. He, X., Zou, W.: Existence and concentration of ground states for Schrödinger-Poisson equations with critical growth J. Math. Phys. 53(2), 143-162 (2012)

14. Lieb, E., Loss, M.: Analysis. Grad. Stud. Math. AMS, Providence (2001)

15. Mauser, N.J.: The Schrödinger-Poisson- $X_{\alpha}$ equation. Appl. Math. Lett. 14, 759-763 (2001)

16. Stubbe, J.: Bound states of two-dimensional Schrödinger-Newton equation (2008). arXiv:0807.4059v1

17. Wen, L., Chen, S., Radulescu, V.D.: Axially symmetric solutions of the Schrödinger-Poisson system with zero mass potential in $\mathbb{R}^{2}$. Appl. Math. Lett. 104, Article ID 106244 (2020)

18. Willem, M.: Minimax Theorems. Progress in Nonlinear Differential Equations and Their Applications, vol. 24. Birkhäuser Boston, Boston (1996)

19. Xu, L., Chen, H.: Ground state solutions for Kirchhoff-type equations with a general nonlinearity in the critical growth. Adv. Nonlinear Anal. 7(4), 535-546 (2018)

20. Zhao, L., Zhao, F.: Positive solutions for Schrödinger-Poisson equations with critical exponent. Nonlinear Anal. 70 , 2150-2164 (2009)

\section{Submit your manuscript to a SpringerOpen ${ }^{\circ}$ journal and benefit from:}

- Convenient online submission

- Rigorous peer review

- Open access: articles freely available online

- High visibility within the field

- Retaining the copyright to your article

Submit your next manuscript at $\boldsymbol{\nabla}$ springeropen.com 